RESEARCH ARTICLE

\title{
An Investigation into the Scale and Impact of Self- Reported Foot Problems Associated with Systemic Lupus Erythematosus: A Study Protocol and Survey Questionnaire Development
}

\author{
Anita Ellen Williams ${ }^{1 *}$, Lindsey Cherry ${ }^{2}$, Alison Blake ${ }^{3}$, Begonya Alcacer-Pitarch ${ }^{4}$, \\ Christopher Edwards ${ }^{2}$, Neil Hopkinson ${ }^{5}$, Edward Vital ${ }^{4}$ \& Lee-Suan Teh ${ }^{6}$ \\ ${ }^{1}$ School of Health Science, University of Salford M6 6PU, UK \\ ${ }^{2}$ Faculty of Health Sciences, University of Southampton Southampton, UK \\ ${ }^{3}$ Great Western Hospitals NHS FT Wiltshire, UK \\ ${ }^{4}$ Leeds Institute of Rheumatic and Musculoskeletal Medicine Leeds, UK \\ ${ }^{5}$ Christchurch Hospital Christchurch, UK \\ ${ }^{6}$ The Royal Blackburn Hospital Blackburn, UK
}

\begin{abstract}
Background. Systemic lupus erythematosus (SLE) can manifest with arthralgia and myalgia, and, in severe cases, disorganization of the joints and tendon rupture. Further, Raynaud's phenomenon and other circulatory problems such as vasculitis have been reported, and may be associated with loss of sensation and ulcers. Associated with impaired peripheral neurovascular function there is the potential for changes in tissue viability leading to thinning of the skin or callus formation. In addition, resistance to infections may be reduced, such as fungal infection of the skin and nails, bacterial infection associated with wounds and viral infections such as verruca. There is a dearth of evidence for the effects of SLE in the foot, the prevalence of foot problems in SLE and the impact of these on the individual. In addition, it is not known if people with SLE and foot problems have access to specialist care through foot health services. Hence, there is a need to investigate the scale of foot problems associated with SLE. In order to achieve this, a questionnaire needs to be developed in order to carry out a national survey in England. Methods. The items required for the questionnaire were generated using a focus group, which comprised patient advisers with SLE, consultants who specialized in SLE, specialist rheumatology podiatrists and specialist rheumatology nurses. From this consensus approach to the item generation, the draft questionnaire was developed with agreement on themes, question format and overall structure. Additionally, the Manchester Pain and Disability Questionnaire was included in order to capture levels of pain and associated disability. An iterative process followed, with feedback from the focus group reducing the number of other items from 53, until the penultimate version of questionnaire was produced with 50 items. Following on from this, a process of cognitive debriefing was used with two people with SLE who were naïve to the questionnaire. Minor changes to two questions and the layout was required before a final version of the questionnaire was produced.

Discussion. The questionnaire will be used for a study which aims to identify the frequency of patients' selfreported foot problems, the impact of foot problems on their lives and the status of foot care provision. This will be achieved through a survey of people with SLE across six clinical sites and interviews with some people in order to explore their experience of foot problems. The results from the present study will provide the information required to inform further research. In addition, it could potentially inform the design and delivery of foot health information and services to this patient group. Copyright $\odot 2015$ John Wiley \& Sons, Ltd.
\end{abstract}

Keywords

Systemic lupus erythematosus; foot; podiatry; questionnaire; interviews 
*Correspondence

Anita Ellen Williams, School of Health Science, University of Salford M6 6PU,UK.

Email: a.e.williams1@salford.ac.uk

Published online in Wiley Online Library (wileyonlinelibrary.com) DOI: 10.1002/msc.1119

\section{Background}

Systemic lupus erythematosus (SLE) is a complex, chronic, multi-system autoimmune disease, which varies in prevalence and incidence depending on ethnicity (D'Cruz et al., 2007; Hopkinson et al., 1993; Lau et al., 2006). It affects more females than males, with a ratio of 9:1 (D'Cruz et al., 2007; Hopkinson et al., 1993; Lau et al., 2006), and in the UK approximately 25,000 people have a diagnosis of SLE (Nightingale et al., 2007). The disease varies in manifestations and severity between individuals but can affect the patients' quality of life, in spite of the control of disease symptoms with disease-modifying drugs (McElhone et al., 2010).

Involvement of the musculoskeletal system is common during the clinical course of SLE in up to $95 \%$ of patients, with joint pain being the first presenting symptom in up to $50 \%$ of cases (Zoma, 2004). The extra articular manifestations of SLE include soft tissue pathology such as capsular swelling, synovial hypertrophy and tenosynovitis (Mukherjee et al., 2014; Ostendorf et al., 2003), which can lead to tendon rupture (Petri, 1995) and tendon contractures (Grigor et al., 1978). Tendons are the forcetransmitting units of the musculoskeletal system but, because of their low metabolic rate and slow healing (Grigor et al., 1978), injury can result in considerable morbidity and prolonged disability. In relation to the symptoms associated with muscle involvement, it can range in severity from mild aches, reported in up to $80 \%$ of cases, to inflammatory myositis, in up to $11 \%$ of cases (Zoma, 2004).

In relation to the effects of SLE on the cardiovascular system, Mathieu et al. (2000) identified that patients with SLE had an increased risk of Raynaud's syndrome. Further to this, early vascular alterations have been identified as being pronounced in SLE, increasing cardiovascular disease risk (Santos et al., 2012) and, compared with the general population, is at least doubled (Schoenfeld et al., 2013). Further,
Esdaile et al. (2001) identified a substantial and statistically significant increase in cardiovascular problems that cannot be fully explained by traditional Framingham risk factors alone. In relation to peripheral arterial disease (PVD) specifically, Bhatt et al. (2007) found that in 50 people with SLE, 62\% had dyslipidaemia, which was found independently to affect the occurrence of PVD.

However, despite these studies indicating musculoskeletal and vascular involvement, a narrative review (Williams et al., 2013) concluded that the number of studies focusing on the lower limb and foot was small. Hence, the scale of these disease-specific problems and how they affect the feet of people with SLE is unknown. Additionally, it could be speculated that common foot complaints that occur in the general population, such as corns and callus, may have more impact in this patient group. To date, there has been no study reporting the frequency and nature of any foot problems in this population or how these affect patients' overall quality of life and experience of living with SLE (McElhone et al., 2010). In other inflammatory rheumatic diseases, such as rheumatoid arthritis (Williams and Graham, 2012) and scleroderma (Alcacer-Pitarch et al., 2011), foot and lower limb disorders have been recognized as under-treated and important to people's perceptions of their overall well-being. Many of the issues in these diseases, such as musculoskeletal and vascular disorders, may apply equally to SLE patients. We therefore proposed to identify the frequency of patients' self-reported foot problems, the impact of foot problems on their lives, and the status of foot care provision. In order to achieve this, three sequential objectives need to be carried out: i) to develop a questionnaire on selfreported foot problems, their impact and foot care provision; ii) to disseminate the questionnaire; and iii) to interview some people with SLE in order to explore their experiences of living with foot problems. 


\section{Methods}

\section{Study design}

The study design was both quantitative (through the survey questionnaire) and qualitative (through free text in the survey questionnaire and patient interviews). Ethical approval was obtained through the Integrated Research Approval System (IRAS) (14/SC/1009) and University of Salford ethics panel (HSCR14/25).

\section{Advisory group}

The study was supported through a core advisory group comprising two people with SLE as patient advisers; the chief investigator; the co-investigator; the research associate; two specialist nurses and two consultant rheumatologists. The purpose of the core advisory group was to act as a steering group in order to monitor progress, review the results of the research and guide the dissemination of the results.

\section{Survey questionnaire development}

The members of the advisory group also formed a survey questionnaire development group. The development of the questionnaire was achieved through a focus-group method (Oppenheim, 2001). The focus group was facilitated by the research associate, with one of the advisory group members taking field notes. The members of the group expressed their own experiences and priorities in relation to foot problems, and from this an item pool was generated. Following this, consensus was achieved for the specific items and themes to be included in the questionnaire (Oppenheim, 2001). It was decided by consensus that the Manchester Foot Pain and Disability Questionnaire (MFPDQ) (Garrow et al., 2000) should be included in order to capture levels of pain and associated disability. In addition to the items in the MFPDQ, the focus group generated 53 items under the headings of seven themes: circulation; nerve supply; skin problems; joints, muscles and tendons; care of feet; feet and work; foot pain; and the participants' demographics. The question format and style, the groupings of questions and the overall structure were agreed. Following two iterations and feedback from the focus group members, three items were removed as they were thought to be repetitive of others and the layout was modified (for example, the themes were formatted in different colours for clarity).
The focus group members checked the foot health survey questionnaire for face and content validity prior to cognitive debriefing (Oppenheim, 2001) with two people with SLE who were naïve to the questionnaire. This process was used in order to ensure usability, understanding of the process of completing the survey and understanding of the questions, prior to its dissemination. Following this process, two questions were modified in order to remove ambiguity. The questionnaire was finalized (with the addition of participant information) and is currently being disseminated to people with SLE.

\section{Questionnaire survey}

Patients will be recruited consecutively from:

- The Royal Blackburn Hospital, Blackburn, UK

- University Hospital Southampton NHS Foundation Trust, Southampton, UK

- The Royal Bournemouth and Christchurch Hospitals NHS Foundation Trust, Bournemouth, UK

- Leeds Teaching Hospitals NHS Trust, Chapel Allerton Hospital, Leeds, UK

- Salford Royal Hospitals Foundation Trust, Salford, UK

- Manchester Royal Infirmary, Manchester, UK

Patients will be invited by their consultant to consider completing the questionnaire and while attending clinic. Patients will be reassured by their consultant that a decision to take part, or not, in the survey, will have no impact on their ongoing care provision.

The patient inclusion criteria will be those patients who are:

1. Diagnosed with SLE, according to American College of Rheumatology (ACR) criteria (Petri et al., 2012; Tan et al., 1982), as the primary diagnosis.

2. Able and agree to complete the self-reported questionnaire on foot/lower limb problems.

3. Aged 18 years or over.

4. Able to read and comprehend the English language.

The patient exclusion criteria will be those patients who:

1. Do not have a definite diagnosis of SLE, according to ACR criteria (Petri et al., 2012; Tan et al., 1982), as the primary diagnosis. 
2. Are not able or willing to complete the self-reported questionnaire on foot/lower limb problems.

3. Less than 18 years of age.

4. Are involved in another research study that would, in the patient or investigators' opinions, conflict with the present one.

5. Unable to read or comprehend the English language with assistance.

From audit data available from these clinical sites, a pool of potential participants is estimated at $n=400$; given a $50 \%$ response rate, this suggests a sample size of approximately 200 participants.

The survey questionnaire will contain an information sheet about the purpose of the study and consent will be explained as being implied by agreeing to complete the questionnaire. Participants will be assured that all data are anonymous and that the only identification will be a code assigned to each location. The patients' clinical scores for disease activity and current disease status will be measured and recorded by the participants' consultant using the British Isles Lupus Assessment Group's disease activity index (BILAG 2004) (Isenberg et al., 2005). This will be collected in order to identify the participants' level of disease as well as individual patients' organ involvement - for example, whether there is cutaneous, musculoskeletal or vascular disease in other organs as this may be useful in understanding pathology in the foot.

It is anticipated that there will be minimal adverse incidents in the present study but by taking part, it may highlight to participants that they have foot problems that may not have been managed to date. The information sheet given to the participants of the survey will explain what to do if they have foot problems, and, if required, a referral will be made to podiatry services in line with local procedures. Participants will also be given written information about additional external sources of support, such as patient-led support via Lupus UK regional groups, if required.

\section{Questionnaire survey: Data analysis}

SPSS software (version 20) (SPSS Inc., Chicago, IL, USA) will be used to undertake the statistical analysis. Descriptive statistics will be used to describe the participant demographics, disease duration/management and survey data. Summary statistics will be checked for each variable and will consist of a measure of central tendency and dispersion - that is, mean or median, and standard deviation or interquartile ranges, for continuous parametric and non-parametric data, respectively. Assessment of normal distribution and outliers will be checked for continuous data. For nominal and ordinal data, the mode will be reported as an indicator of central tendency, and the frequency of distribution across data categories will be reported descriptively. Non-parametric tests will be used for ordinal data. Where facts are reported in the free text boxes, content analysis will be used. Where opinions and emotions are expressed, thematic analysis will be used. Both will be aligned with the quantitative data in order to expand and illuminate the data.

\section{Participant interviews}

The potential participants $(n=12)$ will be identified as a purposive sample by their consultant at one clinical site (The Royal Bournemouth and Christchurch Hospitals NHS Foundation Trust). The sample size of 12 is similar to that in other qualitative studies which have aimed to obtain a depth of information about personal experiences. The inclusion criteria will be the same as for the survey but participants will also agree to participate in an interview and have had experience of foot problems. A participant information sheet will be provided for the potential participant to read before deciding whether to take part in an interview, and they will be assured that declining will not affect their current or future care. They will have two weeks to decide whether or not to take part.

If they agree to be interviewed, they will have the choice of undergoing this at their clinic or in their home. A safety strategy will be put into place to protect the research associate in the latter case. Consent will be obtained and then conversational-style interviews are proposed using an underpinning interpretivistic phenomenological approach to both data collection and analysis (Silverman, 2000). The interviews will be recorded digitally and complemented by field notes. An opening question will be used for all participants: 'Tell me about your experiences of having foot problems?' If necessary, further trigger questions will be used in order to maintain the conversation and the focus on foot problems: 
'How do(es) this/these problem(s) affect you in your daily activities?'

'How do you feel about these problems?'

'Do you understand why they occur?'

'Do you/have you receive(d) treatment for these problems?'

'Do you have any worries about these problems?'

'...what do you think may help with these problems?'

It is anticipated that the interviews will take no longer than one hour. It is recognized that in-depth interviews have the potential to be emotive, and in that respect if participants become upset then they will have the right to cease the interview and, if they choose, to have the data destroyed. The research associate carrying out the interviews will be sensitive to the participants' needs and wishes and will provide immediate support for this. If required, participants will also be given written information about patientled support groups.

\section{Interview data analysis}

Data will be transcribed verbatim and analysed using a thematic framework approach (Attride-Stirling, 2001) by the research team. Themes that have emerged from the analysis of the qualitative data will be supported with exemplars from the transcripts in order to support the truthfulness of the data and illuminate the themes. In addition, the results will be analysed by a second researcher in order to add to the credibility of the analyses. Further, the results will be reviewed by the participants to confirm that they are a true reflection of their dialogue.

All questionnaire results, recorded data and transcriptions from the participant interviews will be retained electronically according to the University of Salford policy on data management. Finally, all data will be presented in a report, with summaries of the quantitative data and themes/exemplars from the participant interviews. A discussion on the results by the advisory group will be followed by conclusions, and recommendations for practice and for future research, and a dissemination plan will be agreed.

\section{Discussion}

We have described a protocol for a study which combines a quantitative and qualitative investigation into self-reported foot problems in people with SLE. We achieved a structured and rigorous approach to the development of a questionnaire which can identify selfreported foot problems, the impact in relation to pain and disability, the effect on occupation, and how these foot problems are managed. We have also described the study protocol in relation to exploring how some participants experience foot problems through in-depth interviews.

The results of the proposed study could be useful in providing the baseline information required to inform further research. As the current study is investigating 'self-reported' foot problems, this may not provide the full picture of foot problems in this patient group. As there is the potential for foot problems generally to be under-reported (Blake et al., 2013), it may be useful to carry out an objective clinical investigation using a structured approach, including the use of validated tools such as the Ankle Brachial Pressure Index for peripheral arterial problems (Bhatt et al., 2007) and The Foot Posture Index for assessment of the musculoskeletal architecture of the foot (Redmond et al., 2006).

Overall, improved understanding of the frequency and impact of foot complications will ultimately underpin the process by which recommendations can be made regarding the type, frequency and nature of foot health care requirements.

\section{Acknowledgements}

We thank Kevin Kennard and Helen Bifield as patient partners, Dr Karen Walker-Bone for advice on the 'occupation' component of the questionnaire, and Professor Ian Bruce and Professor Ariane Herrick for their contribution to the overall study. We are also grateful to The Logres Trust, which provided the funding for this study. The publications plan was agreed with the trustees of the Logres Trust.

\section{REFERENCES}

Alcacer-Pitarch B, Siddle HJ, Buch MH, Emery P, Hashmi F, Redmond AC (2011). Foot health needs in people with systemic sclerosis: An audit of foot health care provision. Clinical Rheumatology 30: 1611-15.

Attride-Stirling J (2001). Thematic networks: An analytic tool for qualitative research. Qualitative Research 1: 385-405.

Bhatt SP, Handa R, Gulati GS, Sharma S, Pandey RM, Aggarwal P, Ramakrishnan L, Shankar S (2007). 
Peripheral vascular disease in systemic lupus erythematosus. Lupus 16: 720-3.

Blake A, Mandy PJ, Stew G (2013). Factors influencing the patient with rheumatoid arthritis in their decision to seek podiatry. Musculoskeletal Care 11: 218-28.

D’Cruz DP, Khamashta MA, Hughes GR (2007). Systemic lupus erythematosus. Lancet 369: 587-96.

Esdaile JM, Abrahamowicz M, Grodzicky T, Li Y, Panaritis C, du Berger R, Côte R, Grover SA, Fortin PR, Clarke AE, Senécal JL (2001). Traditional Framingham risk factors fail to fully account for accelerated atherosclerosis in systemic lupus erythematosus. Arthritis and Rheumatism 44: 2331-7.

Garrow AP, Papageorgiou AC, Silman AJ, Thomas E, Jayson MI, Macfarlane GJ (2000). Development and validation of a questionnaire to assess disabling foot pain. Pain 85: 107-13.

Grigor R, Edmonds J, Lewkonia R, Bresnihan B, Hughes GR (1978). Systemic lupus erythematosus. A prospective analysis. Annals of the Rheumatic Diseases 37: 121-8.

Hopkinson ND, Doherty M, Powell RJ (1993). The prevalence and incidence of systemic lupus erythematosus in Nottingham, UK, 1989-1990. British Journal of Rheumatology 32: 110-15.

Isenberg DA, Rahman A, Allen E, Farewell V, Akil M, Bruce IN, D'Cruz D, Griffiths B, Khamashta M, Maddison P, McHugh N, Snaith M, Teh LS, Yee CS, Zoma A, Gordon C (2005). BILAG 2004. Development and initial validation of an updated version of the British Isles Lupus Assessment Group's disease activity index for patients with systemic lupus erythematosus. Rheumatology (Oxford) 44: 902-6.

Lau CS, Yin G, Mok MY (2006). Ethnic and geographical differences in systemic lupus erythematosus: An overview. Lupus 15: 715-19.

Mathieu A, Sanna G, Passiu G, Cauli A, Piga M (2000). Raynaud's syndrome in systemic lupus erythematosus. Lancet 355: 1102-3.

McElhone K, Abbott J, Gray J, Williams A, Teh LS (2010). Patient perspective of systemic lupus erythematosus in relation to health-related quality of life concepts: A qualitative study. Lupus 19: 1640-7.

Mukherjee S, Cherry L, Bowen C, Culliford D, Arden N, Edwards CJ (2014). The clinical Importance of forefoot inflammation in patients with systemic lupus erythematosus. British Society of Rheumatology. Liverpool: Oxford Univeristy Press.

Nightingale AL, Farmer RD, de Vries CS (2007). Systemic lupus erythematosus prevalence in the UK: Methodological issues when using the General Practice Research Database to estimate frequency of chronic relapsingremitting disease. Pharmacoepidemiology and Drug Safety 16: 144-51.
Oppenheim AN (2001). Questionnaire Design, Interviewing and Attitude Measurement. London: Continuum.

Ostendorf B, Scherer A, Specker C, Mödder U, Schneider M (2003). Jaccoud's arthropathy in systemic lupus erythematosus: Differentiation of deforming and erosive patterns by magnetic resonance imaging. Arthritis and Rheumatism 48: 157-65.

Petri M (1995). Musculoskeletal complications of systemic lupus erythematosus in the Hopkins Lupus Cohort: An update. Arthritis Care and Research 8: 137-45.

Petri M, Orbai AM, Alarcón GS, Gordon C, Merrill JT, Fortin PR, Bruce IN, Isenberg D, Wallace DJ, Nived O, Sturfelt G, Ramsey-Goldman R, Bae SC, Hanly JG, Sánchez-Guerrero J, Clarke A, Aranow C, Manzi S, Urowitz M, Gladman D, Kalunian K, Costner M, Werth VP, Zoma A, Bernatsky S, Ruiz-Irastorza G, Khamashta MA, Jacobsen S, Buyon JP, Maddison P, Dooley MA, van Vollenhoven RF, Ginzler E, Stoll T, Peschken C, Jorizzo JL, Callen JP, Lim SS, Fessler BJ, Inanc M, Kamen DL, Rahman A, Steinsson K, Franks AG, Sigler L, Hameed S, Fang H, Pham N, Brey R, Weisman MH, McGwin G, Magder LS (2012). Derivation and validation of the Systemic Lupus International Collaborating Clinics classification criteria for systemic lupus erythematosus. Arthritis and Rheumatism 64: 2677-86.

Redmond AC, Crosbie J, Ouvrier RA (2006). Development and validation of a novel rating system for scoring standing foot posture: The Foot Posture Index. Clinical Biomechanics 21: 89-98.

Santos MJ, Carmona-Fernandes D, Canhão H, Canas da Silva J, Fonseca JE, Gil V (2012). Early vascular alterations in SLE and RA patients - A step towards understanding the associated cardiovascular risk. PLoS One 7: e44668.

Schoenfeld SR, Kasturi S, Costenbader KH (2013). The epidemiology of atherosclerotic cardiovascular disease among patients with SLE: A systematic review. Seminars in Arthritis and Rheumatism 43: 77-95.

Silverman D (2000). Doing Qualitative Research. London: Sage.

Tan EM, Cohen AS, Fries JF, Masi AT, McShane DJ, Rothfield NF, Schaller JG, Talal N, Winchester RJ (1982). The 1982 revised criteria for the classification of systemic lupus erythematosus. Arthritis and Rheumatism 25: 1271-7.

Williams AE, Crofts G, Teh LS (2013). 'Focus on feet' The effects of systemic lupus erythematosus: A narrative review of the literature. Lupus 22: 1017-23.

Williams AE, Graham AS (2012). 'My feet: Visible, but ignored...' A qualitative study of foot care for people with rheumatoid arthritis. Clinical Rehabilitation 26: 952-9.

Zoma A (2004). Musculoskeletal involvement in systemic lupus erythematosus. Lupus 13: 851-3. 